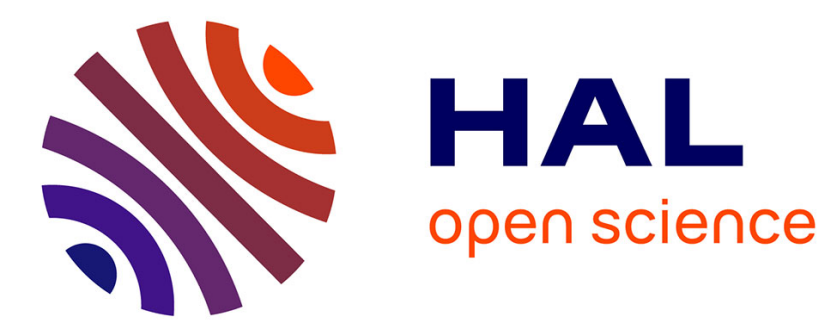

\title{
Well-posedness of a conservation law with non-local flux arising in traffic flow modeling
}

\author{
Sebastien Blandin, Paola Goatin
}

\section{To cite this version:}

Sebastien Blandin, Paola Goatin. Well-posedness of a conservation law with non-local flux arising in traffic flow modeling. Numerische Mathematik, 2016, 10.1007/s00211-015-0717-6 . hal-00954527

\section{HAL Id: hal-00954527 \\ https://hal.inria.fr/hal-00954527}

Submitted on 3 Mar 2014

HAL is a multi-disciplinary open access archive for the deposit and dissemination of scientific research documents, whether they are published or not. The documents may come from teaching and research institutions in France or abroad, or from public or private research centers.
L'archive ouverte pluridisciplinaire HAL, est destinée au dépôt et à la diffusion de documents scientifiques de niveau recherche, publiés ou non, émanant des établissements d'enseignement et de recherche français ou étrangers, des laboratoires publics ou privés. 


\title{
Well-posedness of a conservation law with non-local flux arising in traffic flow modeling
}

\author{
Sebastien Blandin • Paola Goatin
}

Received: date / Accepted: date

\begin{abstract}
We prove the existence and stability of entropy weak solutions of a scalar conservation law with non-local flux arising in traffic flow modeling. The result is obtained providing accurate $\mathbf{L}^{\infty}, B V$ and $\mathbf{L}^{\mathbf{1}}$ estimates for the sequence of approximate solutions constructed by an adapted Lax-Friedrichs scheme.
\end{abstract}

Keywords Scalar conservation laws · Non-local flux · Lax-Friedrichs scheme · Macroscopic Traffic Models

\section{Introduction}

Conservation laws with non-local terms arise in a variety of physical applications. Space-integral terms are considered for example in models for granular flows [1], sedimentation [6], crowd motion [9], or more general problems like gradient constrained equations [2]. Also, non-local in time terms arise in conservation laws with memory, starting from [13].

Macroscopic traffic flow models usually consist of one or two first-order hyperbolic partial differential equations accounting for the conservation of the number of cars, as in the celebrated Lighthill-Whitham-Richards model [19, $20]$, and for a momentum conservation or balance equation, see for example the Aw-Rascle model [4], the general phase transition model [7], or the generalized

This research was partially supported by the European Research Council under the European Union's Seventh Framework Program (FP/2007-2013) / ERC Grant Agreement n. 257661.

\author{
S. Blandin \\ IBM Research Collaboratory, Singapore \\ E-mail: sblandin@sg.ibm.com \\ P. Goatin \\ INRIA Sophia Antipolis - Méditerranée, France \\ E-mail: paola.goatin@inria.fr
}


second order model [17]. The resulting (system of) classical conservation laws usually turns out to be well-posed.

Equations with non-local flux have been recently introduced in traffic flow modeling to account for the reaction of drivers or pedestrians to the surrounding density of other individuals, see $[3,9-12,21]$. While pedestrians are likely to react to the presence of people all around them, drivers will mainly adapt their velocity to the downstream traffic, assigning a greater importance to closer vehicles. In this paper, we consider the following mass conservation equation for traffic flow with non-local mean velocity:

$$
\partial_{t} \rho(t, x)+\partial_{x}\left(\rho(t, x) v\left(\int_{x}^{x+\eta} \rho(t, y) w_{\eta}(y-x) d y\right)\right)=0,
$$

defined for $t \in \mathbb{R}^{+}$and $x \in \mathbb{R}, \eta>0$. Above, the convolution kernel $w_{\eta} \in$ $\mathbf{C}^{\mathbf{1}}\left([0, \eta] ; \mathbb{R}^{+}\right)$is a non-increasing function such that $\int_{0}^{\eta} w_{\eta}(x) d x=1$ (for example, $w_{\eta}(x) \equiv 1 / \eta$ or $\left.w_{\eta}(x)=2(1-x / \eta) / \eta\right)$.

With slight abuse of notation, we define the downstream convolution product as

$$
\rho *_{d} w_{\eta}(t, x):=\int_{x}^{x+\eta} \rho(t, y) w_{\eta}(y-x) d y .
$$

This choice is intended to model the behavior of drivers reacting to what happens in front of them, thus adapting their velocity with respect to the downstream density.

For the sake of simplicity, and aiming to get sharp estimates, in this paper we take the mean velocity function to be $v(r)=1-r$. The same approach could be applied to more general continuous decreasing functions.

Setting $V(t, x)=v\left(\rho *_{d} w_{\eta}(t, x)\right)$, we rewrite (1) as

$$
\partial_{t} \rho(t, x)+\partial_{x}(\rho(t, x) V(t, x))=0,
$$

and we couple it with an initial datum

$$
\rho(0, x)=\rho_{0}(x) \in \mathrm{BV}(\mathbb{R} ;[0,1]) .
$$

We will consider solutions $\rho=\rho(t, x)$ satisfying the following definition.

Definition 1 A function $\rho \in\left(\mathbf{L}^{1} \cap \mathbf{L}^{\infty} \cap \mathrm{BV}\right)\left(\mathbb{R}^{+} \times \mathbb{R} ;[0,1]\right)$ is a weak solution of (1), (3), if

$$
\int_{0}^{+\infty} \int_{-\infty}^{+\infty}\left(\rho \varphi_{t}+\rho(t, x) v\left(\rho *_{d} w_{\eta}\right) \varphi_{x}\right) d x d t+\int_{-\infty}^{+\infty} \rho_{0}(x) \varphi(0, x) d x=0
$$

for all $\varphi \in \mathbf{C}_{\mathbf{c}}^{\mathbf{1}}\left(\mathbb{R}^{2} ; \mathbb{R}\right)$.

Following [16, Definition 1], [6, Definition 4.1] and [10, Definition 2.1], we can also give an entropy criterion to select admissible solutions. 
Definition 2 A function $\rho \in\left(\mathbf{L}^{\mathbf{1}} \cap \mathbf{L}^{\infty} \cap \mathrm{BV}\right)\left(\mathbb{R}^{+} \times \mathbb{R} ;[0,1]\right)$ is an entropy weak solution if

$$
\begin{aligned}
\int_{0}^{+\infty} \int_{-\infty}^{+\infty}\left(|\rho-\kappa| \varphi_{t}+|\rho-\kappa| V \varphi_{x}\right. & \left.-\operatorname{sgn}(\rho-\kappa) k V_{x} \varphi\right)(t, x) d x d t \\
& +\int_{-\infty}^{+\infty}\left|\rho_{0}(x)-\kappa\right| \varphi(0, x) d x \geq 0
\end{aligned}
$$

for all $\varphi \in \mathbf{C}_{\mathbf{c}}^{\mathbf{1}}\left(\mathbb{R}^{2} ; \mathbb{R}^{+}\right)$and $\kappa \in \mathbb{R}$.

We remark that, unlike $[6,10]$, in our case the assumptions of Kružkov are not fully satisfied: the convolution kernel $w_{\eta}$ is not continuous on $\mathbb{R}$. Therefore, uniqueness of the above entropy weak solutions is not guaranteed.

The main results of this paper are collected in the following theorem.

Theorem 1 Let $\rho_{0} \in B V(\mathbb{R} ;[0,1])$ and $w_{\eta} \in \mathbf{C}^{\mathbf{1}}\left([0, \eta] ; \mathbb{R}^{+}\right)$be a non-increasing function such that $\int_{0}^{\eta} w_{\eta}(x) d x=1$. Then the Cauchy problem

$$
\begin{cases}\partial_{t} \rho+\partial_{x}\left(\rho v\left(\rho *_{d} w_{\eta}\right)\right)=0, & x \in \mathbb{R}, t>0, \\ \rho(0, x)=\rho_{0}(x), & x \in \mathbb{R},\end{cases}
$$

admits a weak entropy solution in the sense of Definitions 1 and 2 , such that

$$
\min _{\mathbb{R}}\left\{\rho_{0}\right\} \leq \rho(t, x) \leq \max _{\mathbb{R}}\left\{\rho_{0}\right\}, \quad \text { for a.e. } x \in \mathbb{R}, t>0 .
$$

The existence of a weak entropy solution is proved by constructing a converging sequence of finite volume approximate solutions, defined using an adapted Lax-Friedrichs scheme. Compared to the previous studies [3,6,9-11], our result does not require the kernel $w_{\eta}$ to be smooth, and provides very accurate $\mathbf{L}^{\infty}$ bounds on the solutions (which are given by the maximum principle (6) above). Moreover, equation (1) provides a meaningful model of road traffic evolution. Yet, we can not provide a uniqueness result. Nevertheless, stability estimates issued from the numerical approximation guarantee that the solutions obtained as limit of our finite volume scheme are stable with respect to the initial data in the $\mathbf{L}^{\mathbf{1}}$ norm, see Section 2.4.

The paper is organized as follows. Section 2 contains the description of the finite volume scheme used to construct approximate solutions, and the proofs of its fine properties: maximum principle, bounded total variation, discrete entropy inequalities and $\mathbf{L}^{\mathbf{1}}$ stability estimates. Relying on these results, the proof of Theorem 1 is detailed in Section 3. Some numerical simulations illustrating the properties of the solutions are collected in Section 4 .

\section{A Lax-Friedrichs numerical scheme}

We take a space step $\Delta x$ such that $\eta=N \Delta x$, for some $N \in \mathbb{N}$, and a time step $\Delta t$ subject to a CFL condition which will be specified later. For $j \in \mathbb{Z}$ 
and $n \in \mathbb{N}$, let $x_{j+1 / 2}=j \Delta x$ be the cells interfaces, $x_{j}=(j-1 / 2) \Delta x$ the cells centers and $t^{n}=n \Delta t$ the time mesh. We want to construct a finite volume approximate solution $\rho_{\Delta x}(t, x)=\rho_{j}^{n}$ for $(t, x) \in C_{j}^{n}=\left[t^{n}, t^{n+1}[\times] x_{j-1 / 2}, x_{j+1 / 2}[\right.$.

Let us denote $w_{\eta}^{k}:=w_{\eta}(k \Delta x)$ for $k=0, \ldots, N-1$ and set

$$
V_{j}:=1-\Delta x \sum_{k=0}^{N-1} w_{\eta}^{k} \rho_{j+k},
$$

which involves a quadrature formula to approximate the convolution term. Remark that the above discretization choice for $w_{\eta}$ implies

$$
\Delta x \sum_{k=0}^{N-1} w_{\eta}^{k} \leq 1+w_{\eta}(0) \Delta x .
$$

We consider the following modified Lax-Friedrichs flux adapted to (1):

$$
\begin{aligned}
F_{j+1 / 2}^{n}: & =g\left(\rho_{j}^{n}, \ldots, \rho_{j+N}^{n}\right) \\
& =\frac{1}{2} \rho_{j}^{n} V_{j}^{n}+\frac{1}{2} \rho_{j+1}^{n} V_{j+1}^{n}+\frac{\alpha}{2}\left(\rho_{j}^{n}-\rho_{j+1}^{n}\right),
\end{aligned}
$$

where $\alpha \geq 1$ is the viscosity coefficient. This gives the following $N+2$ points finite volume scheme

$$
\rho_{j}^{n+1}=H\left(\rho_{j-1}^{n}, \ldots, \rho_{j+N}^{n}\right),
$$

where

$$
\begin{aligned}
& H\left(\rho_{j-1}, \ldots, \rho_{j+N}\right) \\
& :=\rho_{j}-\lambda\left(g\left(\rho_{j}, \ldots, \rho_{j+N}\right)-g\left(\rho_{j-1}, \ldots, \rho_{j+N-1}\right)\right) \\
& =\rho_{j}+\frac{\lambda \alpha}{2}\left(\rho_{j-1}-2 \rho_{j}+\rho_{j+1}\right)+\frac{\lambda}{2}\left(\rho_{j-1} V_{j-1}-\rho_{j+1} V_{j+1}\right),
\end{aligned}
$$

with $\lambda=\Delta t / \Delta x$.

It is straightforward to show that $H(\rho, \ldots, \rho)=\rho$ for all $\rho \in[0,1]$ and the numerical flux (9) satisfies the minimal assumptions:

\section{- Consistency:}

$$
g(\rho, \ldots, \rho)=\rho\left(1-\rho \Delta x \sum_{k=0}^{N-1} w_{\eta}^{k}\right) .
$$

In particular, $g(\rho, \ldots, \rho) \rightarrow \rho\left(1-\rho *_{d} w_{\eta}\right)$ as $\Delta x \searrow 0$ for $\rho$ constant.

- Lipschitz continuity: there exists $K>0$ such that

$$
\left|g\left(\rho_{j}, \ldots, \rho_{j+N}\right)-g(\rho, \ldots, \rho)\right| \leq K \max _{0 \leq k \leq N}\left|\rho_{j+k}-\rho\right|
$$

for $\rho, \rho_{j+k} \in[0,1], k=0, \ldots, N$. (8) guarantees that this is is verified for $K=2+\alpha+w_{\eta}(0) \Delta x$. 
Assuming $\rho_{i} \in[0,1]$ for $i=j-1, \ldots, j+N$, straightforward computations give:

$$
\begin{aligned}
\frac{\partial H}{\partial \rho_{j-1}} & =\frac{\lambda}{2}\left(\alpha+1-\Delta x \sum_{k=0}^{N-1} w_{\eta}^{k} \rho_{j+k-1}-\Delta x w_{\eta}^{0} \rho_{j-1}\right) \\
\frac{\partial H}{\partial \rho_{j}} & =1-\lambda\left(\alpha+\frac{1}{2} \Delta x w_{\eta}^{1} \rho_{j-1}\right) \geq 1-\lambda\left(\alpha+\Delta x \frac{w_{\eta}(0)}{2}\right) \\
\frac{\partial H}{\partial \rho_{j+1}} & =\frac{\lambda}{2}\left(\alpha-1+\Delta x \sum_{k=0}^{N-1} w_{\eta}^{k} \rho_{j+k+1}-\Delta x w_{\eta}^{2} \rho_{j-1}+\Delta x w_{\eta}^{0} \rho_{j+1}\right) \\
\frac{\partial H}{\partial \rho_{j+k}} & =\frac{\lambda}{2} \Delta x\left(w_{\eta}^{k-1} \rho_{j+1}-w_{\eta}^{k+1} \rho_{j-1}\right) \\
\frac{\partial H}{\partial \rho_{j+N-1}} & =\frac{\lambda}{2} \Delta x w_{\eta}^{N-2} \rho_{j+1} \\
\frac{\partial H}{\partial \rho_{j+N}} & =\frac{\lambda}{2} \Delta x w_{\eta}^{N-1} \rho_{j+1}
\end{aligned}
$$

Observe that (13e) and (13f) are non-negative. Moreover, the CFL condition

$$
\Delta t \leq \frac{2}{2 \alpha+\Delta x w_{\eta}(0)} \Delta x
$$

ensures the positivity of (13b) and the assumption

$$
\alpha \geq 1+\Delta x w_{\eta}(0)
$$

guarantees the increasing monotonicity w.r.t. $\rho_{j+1}$ in $(13 \mathrm{c})$, and combined with (8), guarantees the non-negativity of (13a). To obtain (14) and (15) we used the fact that $w_{\eta}^{k} \leq w_{\eta}(0)$ for all $k=0, \ldots, N-1$, by non-increasing monotonicity assumption. On the contrary, the sign of (13d) cannot be a-priori determined. (Nevertheless, we observe that if $\rho_{j-1}=\rho_{j+1}$ the derivatives in (13d) are non-negative due to the decreasing monotonicity of $w_{\eta}$.) Therefore, the numerical scheme (9), (10) is not monotone, and classical convergence results do not apply. Nevertheless, we are able to recover the necessary $\mathbf{L}^{\infty}$ and BV bounds.

\subsection{Maximum principle and $\mathbf{L}^{\infty}$ estimates}

The sought $\mathbf{L}^{\infty}$ bound is a direct consequence of a maximum principle property.

Proposition 1 For any initial datum $\rho_{j}^{0}, j \in \mathbb{Z}$, let $\rho_{m}=\min _{j \in \mathbb{Z}}\left\{\rho_{j}^{0}\right\} \in[0,1]$ and $\rho_{M}=\max _{j \in \mathbb{Z}}\left\{\rho_{j}^{0}\right\} \in[0,1]$. Then the finite volume approximation $\rho_{j}^{n}$, $j \in \mathbb{Z}$ and $n \in \mathbb{N}$, constructed using scheme (9), (10) satisfies the bounds

$$
\rho_{m} \leq \rho_{j}^{n} \leq \rho_{M}
$$


for all $j \in \mathbb{Z}$ and $n \in \mathbb{N}$, under the CFL condition (14).

The proof is based on the following lemma.

Lemma 1 Let $0 \leq \rho_{m} \leq \rho_{j}^{n} \leq \rho_{M} \leq 1$ for all $j \in \mathbb{Z}$. Then

$$
\begin{aligned}
H\left(\rho_{m}, \rho_{m}, \rho_{m}, \rho_{j+2}, \ldots, \rho_{j+N-2}, \rho_{m}, \rho_{m}\right) & \geq \rho_{m}, \\
H\left(\rho_{M}, \rho_{M}, \rho_{M}, \rho_{j+2}, \ldots, \rho_{j+N-2}, \rho_{M}, \rho_{M}\right) & \leq \rho_{M} .
\end{aligned}
$$

Proof From (11) we get

$H\left(\rho_{m}, \rho_{m}, \rho_{m}, \rho_{j+2}, \ldots, \rho_{j+N-2}, \rho_{m}, \rho_{m}\right)=\rho_{m}+\frac{\Delta t}{2} \rho_{m} \sum_{k=0}^{N-1} w_{\eta}^{k}\left(\rho_{j+k+1}-\rho_{j+k-1}\right)$,

and we observe that

$$
\begin{aligned}
& \sum_{k=0}^{N-1} w_{\eta}^{k}\left(\rho_{j+k+1}-\rho_{j+k-1}\right) \\
& \quad=\rho_{m}\left[w_{\eta}^{N-2}+w_{\eta}^{N-1}-w_{\eta}^{0}-w_{\eta}^{1}\right]+\sum_{k=1}^{N-2} \rho_{j+k}\left[w_{\eta}^{k-1}-w_{\eta}^{k+1}\right] \\
& \quad \geq \rho_{m}\left[w_{\eta}^{N-2}+w_{\eta}^{N-1}-w_{\eta}^{0}-w_{\eta}^{1}\right]+\rho_{m} \sum_{k=1}^{N-2}\left[w_{\eta}^{k-1}-w_{\eta}^{k+1}\right] \\
& \quad=\rho_{m}\left\{\sum_{k=1}^{N} w_{\eta}^{k-1}-\sum_{k=-1}^{N-2} w_{\eta}^{k+1}\right\}=0
\end{aligned}
$$

where the inequality is due to the non-increasing monotonicity of $w_{\eta}$. Inequality (17) can be recovered following the same procedure.

Proof of Proposition 1 We apply the mean value theorem between the points $R_{j}^{n}=\left(\rho_{j-1}^{n}, \ldots, \rho_{j+N}^{n}\right)$ and

$$
R_{m}^{n}=\left(\rho_{m}, \rho_{m}, \rho_{m}, \rho_{j+2}^{n}, \ldots, \rho_{j+N-2}^{n}, \rho_{m}, \rho_{m}\right),
$$

which by (16) tells us

$$
\begin{aligned}
\rho_{j}^{n+1}=H\left(R_{j}^{n}\right) & =H\left(R_{m}^{n}\right)+\left\langle\nabla H\left(R_{\xi}\right), R_{j}^{n}-R_{m}^{n}\right\rangle \\
& \geq \rho_{m}+\left\langle\nabla H\left(R_{\xi}\right), R_{j}^{n}-R_{m}^{n}\right\rangle,
\end{aligned}
$$

for $R_{\xi}=(1-\xi) R_{m}^{n}+\xi R_{j}^{n}$, for some $\xi \in[0,1]$.

It is now enough to observe that

$$
\frac{\partial H}{\partial \rho_{j+k}}\left(R_{\xi}\right)\left(R_{j}^{n}-R_{m}^{n}\right)_{k}=0 \quad k=2, \ldots, N-2,
$$

since $\left(R_{j}^{n}-R_{m}^{n}\right)_{k}=0$ for $k=2, \ldots, N-2$. Therefore, under the assumptions (14) and (15), we can conclude that $\left\langle\nabla H\left(R_{\xi}\right), R_{j}^{n}-R_{m}^{n}\right\rangle \geq 0$ and therefore by (18) we have proved that

$$
\rho_{j}^{n+1} \geq \rho_{m} .
$$


The upper bound $\rho_{j}^{n+1} \leq \rho_{M}$ is recovered smilarly by considering

$$
R_{M}^{n}=\left(\rho_{M}, \rho_{M}, \rho_{M}, \rho_{j+2}^{n}, \ldots, \rho_{j+N-2}^{n}, \rho_{M}, \rho_{M}\right)
$$

in place of $R_{m}^{n}$ and using (17).

\section{$2.2 \mathrm{BV}$ estimates}

BV estimates cannot be derived here using standard general approaches. Indeed, scheme (10), (11) does not fit the classical assumption of TVD schemes, see [15, Lemma 2.2], and [5] for the generalization of these conditions to $N$ points schemes. Nevertheless, accurate estimates show that the approximate solutions constructed using our numerical scheme have bounded total variation and preserve monotonicity.

Proposition 2 Let $\rho_{0} \in B V(\mathbb{R} ;[0,1])$, and let $\rho_{\Delta x}$ be given by (10), (11). If $\alpha \geq 1+2 \Delta x w_{\eta}(0)$ and the $C F L$ condition $\Delta t \leq 2 \Delta x /\left(2 \alpha+3 \Delta x w_{\eta}(0)\right)$ holds, then for every $T>0$ the following discrete space $B V$ estimate is satisfied

$$
T V\left(\rho_{\Delta x}(T, \cdot)\right) \leq C\left(w_{\eta}, \rho_{0}, T\right):=e^{2 w_{\eta}(0) T} T V\left(\rho_{0}\right) .
$$

In particular, the numerical scheme (10), (11) is monotonicity preserving.

Proof Scheme (10), (11), can be rewritten as

$$
\begin{aligned}
\rho_{j}^{n+1}= & \rho_{j}^{n}-\frac{\lambda}{2}\left(\alpha+1-\Delta x \sum_{k=0}^{N-1} w_{\eta}^{k} \rho_{j+k+1}^{n}-\Delta x w_{\eta}^{0} \rho_{j-1}^{n}\right) \Delta_{j-1 / 2}^{n} \\
& +\frac{\lambda}{2}\left(\alpha-1+\Delta x \sum_{k=0}^{N-1} w_{\eta}^{k} \rho_{j+k+1}^{n}+\Delta x\left(w_{\eta}^{0}+w_{\eta}^{1}\right) \rho_{j-1}^{n}\right) \Delta_{j+1 / 2}^{n} \\
& +\frac{\lambda}{2} \rho_{j-1}^{n} \Delta x \sum_{k=2}^{N-1}\left(w_{\eta}^{k-1}+w_{\eta}^{k}\right) \Delta_{j+k-1 / 2}^{n} \\
& +\frac{\lambda}{2} \Delta x w_{\eta}^{N-1} \rho_{j-1}^{n} \Delta_{j+N-1 / 2}^{n},
\end{aligned}
$$

where we have set $\Delta_{j+k-1 / 2}^{n}=\rho_{j+k}^{n}-\rho_{j+k-1}^{n}$ for $k=0, \ldots, N$.

In the same way we get

$$
\begin{aligned}
\rho_{j+1}^{n+1}= & \rho_{j+1}^{n}-\frac{\lambda}{2}\left(\alpha+1-\Delta x \sum_{k=0}^{N-1} w_{\eta}^{k} \rho_{j+k+2}^{n}-\Delta x w_{\eta}^{0} \rho_{j}^{n}\right) \Delta_{j+1 / 2}^{n} \\
& +\frac{\lambda}{2}\left(\alpha-1+\Delta x \sum_{k=0}^{N-1} w_{\eta}^{k} \rho_{j+k+2}^{n}+\Delta x\left(w_{\eta}^{0}+w_{\eta}^{1}\right) \rho_{j}^{n}\right) \Delta_{j+3 / 2}^{n} \\
& +\frac{\lambda}{2} \rho_{j}^{n} \Delta x \sum_{k=2}^{N-1}\left(w_{\eta}^{k-1}+w_{\eta}^{k}\right) \Delta_{j+k+1 / 2}^{n} \\
& +\frac{\lambda}{2} \Delta x w_{\eta}^{N-1} \rho_{j}^{n} \Delta_{j+N+1 / 2}^{n}
\end{aligned}
$$


Therefore computing the difference gives

$$
\begin{aligned}
& \Delta_{j+1 / 2}^{n+1}= \\
= & \frac{\lambda}{2}\left(\alpha+1-\Delta x \sum_{k=0}^{N-1} w_{\eta}^{k} \rho_{j+k+1}^{n}-\Delta x w_{\eta}^{0} \rho_{j-1}^{n}\right) \Delta_{j-1 / 2}^{n} \\
& +\left[1-\frac{\lambda}{2}\left(2 \alpha-\Delta x w_{\eta}^{0} \rho_{j}^{n}+\Delta x\left(w_{\eta}^{0}+w_{\eta}^{1}\right) \rho_{j-1}^{n}-\Delta x \sum_{k=0}^{N-1} w_{\eta}^{k} \Delta_{j+k+3 / 2}\right)\right] \Delta_{j+1 / 2}^{n} \\
& +\frac{\lambda}{2}\left(\alpha-1+\Delta x\left(w_{\eta}^{0}+w_{\eta}^{1}\right) \rho_{j}^{n}-\Delta x\left(w_{\eta}^{1}+w_{\eta}^{2}\right) \rho_{j-1}^{n}+\Delta x \sum_{k=0}^{N-1} w_{\eta}^{k} \rho_{j+k+2}^{n}\right) \Delta_{j+3 / 2}^{n} \\
& +\frac{\lambda}{2} \Delta x \sum_{k=2}^{N-2}\left[\left(w_{\eta}^{k-1}+w_{\eta}^{k}\right) \rho_{j}^{n}-\left(w_{\eta}^{k}+w_{\eta}^{k+1}\right) \rho_{j-1}^{n}\right] \Delta_{j+k+1 / 2}^{n} \\
& +\frac{\lambda}{2} \Delta x\left[\left(w_{\eta}^{N-2}+w_{\eta}^{N-1}\right) \rho_{j}^{n}-w_{\eta}^{N-1} \rho_{j-1}^{n}\right] \Delta_{j+N-1 / 2}^{n} \\
& +\frac{\lambda}{2} \Delta x w_{\eta}^{N-1} \rho_{j}^{n} \Delta_{j+N+1 / 2}^{n} \cdot
\end{aligned}
$$

Adding and subtracting $\left(w_{\eta}^{k}+w_{\eta}^{k+1}\right) \rho_{j}^{n}$ in the fourth term of the above righthand side, and noting that

$\left[\left(w_{\eta}^{N-2}+w_{\eta}^{N-1}\right) \rho_{j}^{n}-w_{\eta}^{N-1} \rho_{j-1}^{n}\right] \Delta_{j+N-1 / 2}^{n}=\left[w_{\eta}^{N-2} \rho_{j}^{n}+w_{\eta}^{N-1} \Delta_{j-1 / 2}^{n}\right] \Delta_{j+N-1 / 2}^{n}$,

we get

$$
\begin{aligned}
& \Delta_{j+1 / 2}^{n+1}= \\
= & \frac{\lambda}{2}\left(\alpha+1-\Delta x w_{\eta}^{0}\left(\rho_{j-1}^{n}+\rho_{j+1}^{n}\right)-\Delta x\left(w_{\eta}^{1}+w_{\eta}^{2}\right) \rho_{j+2}^{n}-\Delta x \sum_{k=2}^{N-2} w_{\eta}^{k+1} \rho_{j+k}^{n}\right) \Delta_{j-1 / 2}^{n} \\
& +\left[1-\frac{\lambda}{2}\left(2 \alpha-\Delta x w_{\eta}^{0} \rho_{j}^{n}+\Delta x\left(w_{\eta}^{0}+w_{\eta}^{1}\right) \rho_{j-1}^{n}-\Delta x \sum_{k=0}^{N-1} w_{\eta}^{k} \Delta_{j+k+3 / 2}\right)\right] \Delta_{j+1 / 2}^{n} \\
& +\frac{\lambda}{2}\left(\alpha-1+\Delta x\left(w_{\eta}^{0}+w_{\eta}^{1}\right) \rho_{j}^{n}-\Delta x\left(w_{\eta}^{1}+w_{\eta}^{2}\right) \rho_{j-1}^{n}+\Delta x \sum_{k=0}^{N-1} w_{\eta}^{k} \rho_{j+k+2}^{n}\right) \Delta_{j+3 / 2}^{n} \\
& +\frac{\lambda}{2} \rho_{j}^{n} \Delta x \sum_{k=2}^{N-2}\left(w_{\eta}^{k-1}-w_{\eta}^{k+1}\right) \Delta_{j+k+1 / 2}^{n} \\
& +\frac{\lambda}{2} \Delta x w_{\eta}^{N-2} \rho_{j}^{n} \Delta_{j+N-1 / 2}^{n}+\frac{\lambda}{2} \Delta x w_{\eta}^{N-1} \rho_{j}^{n} \Delta_{j+N+1 / 2}^{n} .
\end{aligned}
$$

Observe that the first coefficient in the summation is non-negative for $\Delta x$ sufficiently small such that $\alpha \geq 2 w_{\eta}(0) \Delta x$, since

$$
\begin{aligned}
& \Delta x w_{\eta}^{0}\left(\rho_{j-1}^{n}+\rho_{j+1}^{n}\right)+\Delta x\left(w_{\eta}^{1}+w_{\eta}^{2}\right) \rho_{j+2}^{n}+\Delta x \sum_{k=2}^{N-2} w_{\eta}^{k+1} \rho_{j+k}^{n} \\
& \leq \Delta x w_{\eta}^{0}+\Delta x \sum_{k=0}^{N-1} w_{\eta}^{k} \\
& \leq 1+2 w_{\eta}(0) \Delta x
\end{aligned}
$$


by (8). The second coefficient in the summation is non-negative under the slightly stronger CFL assumption

$$
\Delta t \leq \frac{2}{2 \alpha+3 \Delta x w_{\eta}(0)} \Delta x,
$$

and the third term is non-negative if $\alpha \geq 1+2 \Delta x w_{\eta}(0)$. Hence all the above coefficients are non-negative, and the above formula guarantees in particular that the scheme (10), (11) is monotonicity preserving. Taking the absolute values in the above expression and summing over $j \in \mathbb{Z}$ we get

$$
\begin{aligned}
& \sum_{j}\left|\Delta_{j+1 / 2}^{n+1}\right|= \\
= & \sum_{j} \frac{\lambda}{2}\left(\alpha+1-\Delta x w_{\eta}^{0}\left(\rho_{j-1}^{n}+\rho_{j+1}^{n}\right)-\Delta x\left(w_{\eta}^{1}+w_{\eta}^{2}\right) \rho_{j+2}^{n}-\Delta x \sum_{k=2}^{N-2} w_{\eta}^{k+1} \rho_{j+k}^{n}\right)\left|\Delta_{j-1 / 2}^{n}\right| \\
& +\sum_{j}\left[1-\frac{\lambda}{2}\left(2 \alpha-\Delta x w_{\eta}^{0} \rho_{j}^{n}+\Delta x\left(w_{\eta}^{0}+w_{\eta}^{1}\right) \rho_{j-1}^{n}-\Delta x \sum_{k=0}^{N-1} w_{\eta}^{k} \Delta_{j+k+3 / 2}\right)\right]\left|\Delta_{j+1 / 2}^{n}\right| \\
& +\sum_{j} \frac{\lambda}{2}\left(\alpha-1+\Delta x\left(w_{\eta}^{0}+w_{\eta}^{1}\right) \rho_{j}^{n}-\Delta x\left(w_{\eta}^{1}+w_{\eta}^{2}\right) \rho_{j-1}^{n}+\Delta x \sum_{k=0}^{N-1} w_{\eta}^{k} \rho_{j+k+2}^{n}\right)\left|\Delta_{j+3 / 2}^{n}\right| \\
& +\sum_{j} \frac{\lambda}{2} \rho_{j}^{n} \Delta x \sum_{k=2}^{N-2}\left(w_{\eta}^{k-1}-w_{\eta}^{k+1}\right)\left|\Delta_{j+k+1 / 2}^{n}\right| \\
& +\sum_{j} \frac{\lambda}{2} \Delta x w_{\eta}^{N-2} \rho_{j}^{n}\left|\Delta_{j+N-1 / 2}^{n}\right| \\
& +\sum_{j} \frac{\lambda}{2} \Delta x w_{\eta}^{N-1} \rho_{j}^{n}\left|\Delta_{j+N+1 / 2}^{n}\right| .
\end{aligned}
$$

Rearranging the indices we obtain

$$
\begin{aligned}
\sum_{j}\left|\Delta_{j+1 / 2}^{n+1}\right| & \\
= & \sum_{j}\left|\Delta_{j+1 / 2}^{n}\right|\left[1+\frac{\Delta t}{2}\left(\sum_{k=2}^{N-2}\left(w_{\eta}^{k-1}-w_{\eta}^{k+1}\right) \rho_{j+k+1}^{n}-\left(w_{\eta}^{1}+w_{\eta}^{2}\right) \rho_{j-2}^{n}\right.\right. \\
& +\sum_{k=2}^{N-2}\left(w_{\eta}^{k-1}-w_{\eta}^{k+1}\right) \rho_{j-k}^{n}-\left(w_{\eta}^{1}+w_{\eta}^{2}\right) \rho_{j+3}^{n}+w_{\eta}^{N-2} \rho_{j+N}^{n} \\
& \left.\left.+w_{\eta}^{N-1} \rho_{j+N+1}^{n}+w_{\eta}^{N-2} \rho_{j-N-1}^{n}+w_{\eta}^{N-1} \rho_{j-N}^{n}\right)\right] \\
\leq & {\left[1+\frac{\Delta t}{2}\left(2 \sum_{k=2}^{N-2}\left(w_{\eta}^{k-1}-w_{\eta}^{k+1}\right)+2 w_{\eta}^{N-2}+2 w_{\eta}^{N-1}\right)\right] \sum_{j}\left|\Delta_{j+1 / 2}^{n}\right| } \\
\leq & \left(1+2 w_{\eta}(0) \Delta t\right) \sum_{j}\left|\Delta_{j+1 / 2}^{n}\right|
\end{aligned}
$$


Therefore we recover the following estimate for the total variation

$$
\operatorname{TV}\left(\rho_{\Delta x}(T, \cdot)\right) \leq\left(1+2 w_{\eta}(0) \Delta t\right)^{T / \Delta t} \operatorname{TV}\left(\rho_{\Delta x}(0, \cdot)\right) \leq e^{2 w_{\eta}(0) T} \operatorname{TV}\left(\rho_{0}\right) .
$$

From Proposition 2, the following space-time BV estimate can be derived as in [14, Corollary 5.1], relying on the Lipschitz continuity of $g$.

Corollary 1 Let $\rho_{0} \in B V(\mathbb{R} ;[0,1])$, and let $\rho_{\Delta x}$ be given by (10), (11). If $\alpha \geq 1+2 \Delta x w_{\eta}(0)$ and $\Delta t \leq 2 \Delta x /\left(2 \alpha+3 \Delta x w_{\eta}(0)\right)$, then for every $T>0$ there exists $\widetilde{C}=\widetilde{C}\left(w_{\eta}, \rho_{0}, T, \alpha\right)$ such that

$$
T V\left(\rho_{\Delta x} ;[0, T] \times \mathbb{R}\right) \leq \widetilde{C} .
$$

2.3 Discrete entropy inequalities

Following [3, Proposition 2.8], we derive a discrete entropy inequality for the approximate solution generate by (10), (11). Let us denote by

$$
\begin{aligned}
G_{j+1 / 2}(u, v) & =\frac{1}{2} u V_{j}^{n}+\frac{1}{2} v V_{j+1}^{n}+\frac{\alpha}{2}(u-v), \\
F_{j+1 / 2}^{\kappa}(u, v) & =G_{j+1 / 2}(u \wedge \kappa, v \wedge \kappa)-G_{j+1 / 2}(u \vee \kappa, v \vee \kappa),
\end{aligned}
$$

with $a \wedge b=\max (a, b)$ and $a \vee b=\min (a, b)$.

Proposition 3 Let $\rho_{j}^{n}, j \in \mathbb{Z}, n \in \mathbb{N}$, be given by (9), (10) Then, if $\alpha \geq 1$ and the CFL condition (14) holds, for all $j \in \mathbb{Z}, n \in \mathbb{N}$ we have

$$
\begin{aligned}
\left|\rho_{j}^{n+1}-\kappa\right|-\left|\rho_{j}^{n}-\kappa\right|+\lambda\left(F_{j+1 / 2}^{\kappa}\left(\rho_{j}^{n}, \rho_{j+1}^{n}\right)-F_{j-1 / 2}^{\kappa}\left(\rho_{j-1}^{n}, \rho_{j}^{n}\right)\right) & \\
+ & \frac{\lambda}{2} \operatorname{sgn}\left(\rho_{j}^{n+1}-\kappa\right) \kappa\left(V_{j+1}^{n}-V_{j-1}^{n}\right) \leq 0
\end{aligned}
$$

for all $\kappa \in \mathbb{R}$.

Proof Setting

$$
\tilde{H}_{j}(u, v, z)=v-\lambda\left(G_{j+1 / 2}(v, z)-G_{j-1 / 2}(u, v)\right),
$$

the function $\tilde{H}_{j}$ is monotone non-decreasing in its first variable, monotone non-decreasing in its second variable for $\alpha \lambda \leq 1$, which is guaranteed by the CFL condition (14), and monotone non-decreasing in its third variable for $\alpha \geq 1$, which is guaranteed by (15). Moreover, we have the identity

$$
\begin{aligned}
\tilde{H}_{j}\left(\rho_{j-1}^{n} \wedge \kappa, \rho_{j}^{n} \wedge \kappa, \rho_{j+1}^{n} \wedge \kappa\right)-\tilde{H}_{j}\left(\rho_{j-1}^{n} \vee \kappa, \rho_{j}^{n} \vee \kappa, \rho_{j+1}^{n} \vee \kappa\right) \\
=\left|\rho_{j}^{n}-\kappa\right|-\lambda\left(F_{j+1 / 2}^{\kappa}\left(\rho_{j}^{n}, \rho_{j+1}^{n}\right)-F_{j-1 / 2}^{\kappa}\left(\rho_{j-1}^{n}, \rho_{j}^{n}\right)\right) .
\end{aligned}
$$


By monotonicity,

$$
\begin{aligned}
\tilde{H}_{j}\left(\rho_{j-1}^{n} \wedge \kappa, \rho_{j}^{n} \wedge \kappa, \rho_{j+1}^{n} \wedge \kappa\right)-\tilde{H}_{j}\left(\rho_{j-1}^{n} \vee \kappa, \rho_{j}^{n} \vee \kappa, \rho_{j+1}^{n} \vee \kappa\right) \\
=\tilde{H}_{j}\left(\rho_{j-1}^{n}, \rho_{j}^{n}, \rho_{j+1}^{n}\right) \wedge \tilde{H}_{j}(\kappa, \kappa, \kappa)-\tilde{H}_{j}\left(\rho_{j-1}^{n}, \rho_{j}^{n}, \rho_{j+1}^{n}\right) \vee \tilde{H}_{j}(\kappa, \kappa, \kappa) \\
=\left|\tilde{H}_{j}\left(\rho_{j-1}^{n}, \rho_{j}^{n}, \rho_{j+1}^{n}\right)-\tilde{H}_{j}(\kappa, \kappa, \kappa)\right| \\
=\operatorname{sgn}\left(\tilde{H}_{j}\left(\rho_{j-1}^{n}, \rho_{j}^{n}, \rho_{j+1}^{n}\right)-\tilde{H}_{j}(\kappa, \kappa, \kappa)\right) \times\left(\tilde{H}_{j}\left(\rho_{j-1}^{n}, \rho_{j}^{n}, \rho_{j+1}^{n}\right)-\tilde{H}_{j}(\kappa, \kappa, \kappa)\right) \\
=\operatorname{sgn}\left(\tilde{H}_{j}\left(\rho_{j-1}^{n}, \rho_{j}^{n}, \rho_{j+1}^{n}\right)-\kappa+\frac{\lambda}{2} \kappa\left(V_{j+1}^{n}-V_{j-1}^{n}\right)\right) \\
\quad \times\left(\tilde{H}_{j}\left(\rho_{j-1}^{n}, \rho_{j}^{n}, \rho_{j+1}^{n}\right)-\kappa+\frac{\lambda}{2} \kappa\left(V_{j+1}^{n}-V_{j-1}^{n}\right)\right) \\
\geq \operatorname{sgn}\left(\tilde{H}_{j}\left(\rho_{j-1}^{n}, \rho_{j}^{n}, \rho_{j+1}^{n}\right)-\kappa\right) \times\left(\tilde{H}_{j}\left(\rho_{j-1}^{n}, \rho_{j}^{n}, \rho_{j+1}^{n}\right)-\kappa+\frac{\lambda}{2} \kappa\left(V_{j+1}^{n}-V_{j-1}^{n}\right)\right) \\
=\left|\tilde{H}_{j}\left(\rho_{j-1}^{n}, \rho_{j}^{n}, \rho_{j+1}^{n}\right)-\kappa\right|+\frac{\lambda}{2} \operatorname{sgn}\left(\tilde{H}_{j}\left(\rho_{j-1}^{n}, \rho_{j}^{n}, \rho_{j+1}^{n}\right)-\kappa\right) \kappa\left(V_{j+1}^{n}-V_{j-1}^{n}\right) \\
=\left|\rho_{j}^{n+1}-\kappa\right|+\frac{\lambda}{2} \operatorname{sgn}\left(\rho_{j}^{n+1}-\kappa\right) \kappa\left(V_{j+1}^{n}-V_{j-1}^{n}\right),
\end{aligned}
$$

by definition of the scheme (10), (11), which gives (21).

\section{$2.4 \mathbf{L}^{\mathbf{1}}$ stability estimates}

Since Kružkov theory cannot be applied as it is to our case, because of lack of regularity of the convolution kernel $w_{\eta}$, we prove explicit $\mathbf{L}^{\mathbf{1}}$ estimates that ensure the stability of the scheme (10), (11).

Proposition 4 Let $\rho_{0}, \bar{\rho}_{0} \in B V(\mathbb{R} ;[0,1])$ be two initial data, and denote by $\rho_{\Delta x}, \bar{\rho}_{\Delta x}$ the corresponding approximate solutions constructed applying the modified Lax-Friedrichs scheme (10), (11):

$$
\begin{aligned}
& \rho_{j}^{n+1}=\rho_{j}^{n}+\frac{\lambda \alpha}{2}\left(\rho_{j-1}^{n}-2 \rho_{j}^{n}+\rho_{j+1}^{n}\right)+\frac{\lambda}{2}\left(\rho_{j-1}^{n} V_{j-1}^{n}-\rho_{j+1} V_{j+1}^{n}\right), \\
& \bar{\rho}_{j}^{n+1}=\bar{\rho}_{j}^{n}+\frac{\lambda \alpha}{2}\left(\bar{\rho}_{j-1}^{n}-2 \bar{\rho}_{j}^{n}+\bar{\rho}_{j+1}^{n}\right)+\frac{\lambda}{2}\left(\bar{\rho}_{j-1}^{n} \bar{V}_{j-1}^{n}-\bar{\rho}_{j+1} \bar{V}_{j+1}^{n}\right)
\end{aligned}
$$

where we have set $V_{j}^{n}=1-\Delta x \sum_{k=0}^{N-1} w_{\eta}^{k} \rho_{j+k}^{n}$ and $\bar{V}_{j}^{n}=1-\Delta x \sum_{k=0}^{N-1} w_{\eta}^{k} \bar{\rho}_{j+k}^{n}$. Then, under assumptions (14) and (15), the following estimate holds:

$$
\left\|\rho_{\Delta x}(T, \cdot)-\bar{\rho}_{\Delta x}(T, \cdot)\right\|_{\mathbf{L}^{1}} \leq K\left(w_{\eta}, \rho_{0}, \bar{\rho}_{0}, T\right)\left\|\rho_{0}-\bar{\rho}_{0}\right\|_{\mathbf{L}^{1}}
$$

with $K\left(w_{\eta}, \rho_{0}, \bar{\rho}_{0}, T\right):=\exp \left(T w_{\eta}(0)\left(1+0.5 \min \left\{C\left(w_{\eta}, \rho_{0}, T\right), C\left(w_{\eta}, \bar{\rho}_{0}, T\right)\right\}\right)\right)$. 
Proof Subtracting (23) from (22) we get

$$
\begin{aligned}
\rho_{j}^{n+1} & -\bar{\rho}_{j}^{n+1}= \\
= & (1-\lambda \alpha)\left(\rho_{j}^{n}-\bar{\rho}_{j}^{n}\right)+\frac{\lambda \alpha}{2}\left(\rho_{j-1}^{n}-\bar{\rho}_{j-1}^{n}\right)+\frac{\lambda \alpha}{2}\left(\rho_{j+1}^{n}-\bar{\rho}_{j+1}^{n}\right) \\
& +\frac{\lambda}{2}\left(\rho_{j-1}^{n}\left(V_{j-1}^{n}-\bar{V}_{j-1}^{n}\right)+\left(\rho_{j-1}^{n}-\bar{\rho}_{j-1}^{n}\right) \bar{V}_{j-1}^{n}\right) \\
& -\frac{\lambda}{2}\left(\left(\rho_{j+1}^{n}-\bar{\rho}_{j+1}^{n}\right) \bar{V}_{j+1}^{n}+\rho_{j+1}^{n}\left(V_{j+1}^{n}-\bar{V}_{j+1}^{n}\right)\right) \\
= & \left(1-\lambda \alpha-\frac{\lambda}{2} \Delta x w_{\eta}^{1} \rho_{j-1}^{n}\right)\left(\rho_{j}^{n}-\bar{\rho}_{j}^{n}\right) \\
& +\frac{\lambda}{2}\left(\alpha+\bar{V}_{j-1}^{n}-\Delta x w_{\eta}^{0} \rho_{j-1}^{n}\right)\left(\rho_{j-1}^{n}-\bar{\rho}_{j-1}^{n}\right) \\
& +\frac{\lambda}{2}\left(\alpha-\bar{V}_{j+1}^{n}+\Delta x w_{\eta}^{0} \rho_{j+1}^{n}-\Delta x w_{\eta}^{2} \rho_{j-1}^{n}\right)\left(\rho_{j+1}^{n}-\bar{\rho}_{j+1}^{n}\right) \\
& +\frac{\lambda}{2} \Delta x \sum_{k=2}^{N-2}\left(w_{\eta}^{k-1} \rho_{j+1}^{n}-w_{\eta}^{k+1} \rho_{j-1}^{n}\right)\left(\rho_{j+k}^{n}-\bar{\rho}_{j+k}^{n}\right) \\
& +\frac{\lambda}{2} \Delta x w_{\eta}^{N-2} \rho_{j+1}^{n}\left(\rho_{j+N-1}^{n}-\bar{\rho}_{j+N-1}^{n}\right)+\frac{\lambda}{2} \Delta x w_{\eta}^{N-1} \rho_{j+1}^{n}\left(\rho_{j+N}^{n}-\bar{\rho}_{j+N}^{n}\right) .
\end{aligned}
$$

Observe that the coefficient of the first term is positive thanks to (14) and the coefficients of the second and third terms are positive thanks to (15). Therefore, taking the absolute values in the above equality we get

$$
\begin{aligned}
& \left|\rho_{j}^{n+1}-\bar{\rho}_{j}^{n+1}\right| \leq \\
\leq & \left(1-\lambda \alpha-\frac{\lambda}{2} \Delta x w_{\eta}^{1} \rho_{j-1}^{n}\right)\left|\rho_{j}^{n}-\bar{\rho}_{j}^{n}\right| \\
& +\frac{\lambda}{2}\left(\alpha+\bar{V}_{j-1}^{n}-\Delta x w_{\eta}^{0} \rho_{j-1}^{n}\right)\left|\rho_{j-1}^{n}-\bar{\rho}_{j-1}^{n}\right| \\
& +\frac{\lambda}{2}\left(\alpha-\bar{V}_{j+1}^{n}+\Delta x w_{\eta}^{0} \rho_{j+1}^{n}-\Delta x w_{\eta}^{2} \rho_{j-1}^{n}\right)\left|\rho_{j+1}^{n}-\bar{\rho}_{j+1}^{n}\right| \\
& +\frac{\lambda}{2} \Delta x \sum_{k=2}^{N-2}\left|w_{\eta}^{k-1} \rho_{j+1}^{n}-w_{\eta}^{k+1} \rho_{j-1}^{n}\right|\left|\rho_{j+k}^{n}-\bar{\rho}_{j+k}^{n}\right| \\
& +\frac{\lambda}{2} \Delta x w_{\eta}^{N-2} \rho_{j+1}^{n}\left|\rho_{j+N-1}^{n}-\bar{\rho}_{j+N-1}^{n}\right|+\frac{\lambda}{2} \Delta x w_{\eta}^{N-1} \rho_{j+1}^{n}\left|\rho_{j+N}^{n}-\bar{\rho}_{j+N}^{n}\right| .
\end{aligned}
$$

Summing over $j \in \mathbb{Z}$, rearranging the indexes and observing that by monotonicity of $w_{\eta}$ and using the triangular inequality

$$
\left|w_{\eta}^{k-1} \rho_{j+1}^{n}-w_{\eta}^{k+1} \rho_{j-1}^{n}\right| \leq\left(w_{\eta}^{k-1}-w_{\eta}^{k+1}\right) \rho_{j+1}^{n}+w_{\eta}^{k+1}\left|\rho_{j+1}^{n}-\rho_{j-1}^{n}\right|
$$


we get

$$
\begin{aligned}
\sum_{j}\left|\rho_{j}^{n+1}-\bar{\rho}_{j}^{n+1}\right| \leq & {\left[1+\frac{\Delta t}{2}\left(\sum_{k=2}^{N-2}\left(w_{\eta}^{k-1}-w_{\eta}^{k+1}\right)+w_{\eta}^{N-2}+w_{\eta}^{N-1}\right)\right.} \\
& \left.+\frac{\Delta t}{2} \sum_{k=2}^{N-2} w_{\eta}^{k+1}\left|\rho_{j-k+1}^{n}-\rho_{j-k-1}^{n}\right|\right] \sum_{j}\left|\rho_{j}^{n}-\bar{\rho}_{j}^{n}\right| .
\end{aligned}
$$

Therefore,

$\left.\left\|\rho_{\Delta x}(T, \cdot)-\bar{\rho}_{\Delta x}(T, \cdot)\right\|_{\mathbf{L}^{1}} \leq\left(1+\Delta t \frac{2+\mathrm{TV}\left(\rho_{\Delta x}(T)\right)}{2} w_{\eta}(0)\right)\right)^{T / \Delta t}\left\|\rho_{0}-\bar{\rho}_{0}\right\|_{\mathbf{L}^{1}}$,

which gives the desired estimate (24).

\section{Proof of Theorem 1}

We apply the classical procedure of Lax-Wendroff theorem, see [14, Theorem $5.3]$ and [18, Theorem 12.1], to show that the scheme (10), (11), converges to a weak solution of (1), in the sense of Definition 1.

By Proposition 1 and Corollary 1, we can apply Helly's theorem, stating that there exists a subsequence, still denoted by $\rho_{\Delta x}$, that converges to some $\rho \in\left(\mathbf{L}^{1} \cap \mathbf{L}^{\infty} \cap \mathrm{BV}\right)\left(\mathbb{R}^{+} \times \mathbb{R} ;[0,1]\right)$ in the $\mathbf{L}_{\text {loc }}^{1}$-norm.

Let $\varphi \in \mathbf{C}_{\mathbf{c}}^{\mathbf{1}}\left(\mathbb{R}^{2}\right)$ and multiply (10) by $\varphi\left(t^{n}, x_{j}\right)$. Summing over $j \in \mathbb{Z}$ and $n \in \mathbb{N}$ we get

$$
\begin{aligned}
& \sum_{n} \sum_{j} \varphi\left(t^{n}, x_{j}\right)\left(\rho_{j}^{n+1}-\rho_{j}^{n}\right) \\
& =-\lambda \sum_{n} \sum_{j} \varphi\left(t^{n}, x_{j}\right)\left(g\left(\rho_{j}^{n}, \ldots, \rho_{j+N}^{n}\right)-g\left(\rho_{j-1}^{n}, \ldots, \rho_{j+N-1}^{n}\right)\right) .
\end{aligned}
$$

Summing by parts we obtain

$$
\begin{aligned}
\sum_{j} \varphi\left(0, x_{j}\right) \rho_{j}^{0} & +\sum_{n} \sum_{j}\left(\varphi\left(t^{n}, x_{j}\right)-\varphi\left(t^{n-1}, x_{j}\right)\right) \rho_{j}^{n} \\
& +\lambda \sum_{n} \sum_{j}\left(\varphi\left(t^{n}, x_{j+1}\right)-\varphi\left(t^{n}, x_{j}\right)\right) g\left(\rho_{j}^{n}, \ldots, \rho_{j+N}^{n}\right)=0 .
\end{aligned}
$$

Then we multiply (25) by $\Delta x$ getting

$$
\begin{aligned}
& \Delta x \sum_{j} \varphi\left(0, x_{j}\right) \rho_{j}^{0}+\Delta x \Delta t \sum_{n} \sum_{j} \frac{\varphi\left(t^{n}, x_{j}\right)-\varphi\left(t^{n-1}, x_{j}\right)}{\Delta t} \rho_{j}^{n} \\
& +\Delta x \Delta t \sum_{n} \sum_{j} \frac{\varphi\left(t^{n}, x_{j+1}\right)-\varphi\left(t^{n}, x_{j}\right)}{\Delta x} g\left(\rho_{j}^{n}, \ldots, \rho_{j+N}^{n}\right)=0 .
\end{aligned}
$$


By strong $\mathbf{L}_{\text {loc }}^{1}$ convergence of $\rho_{\Delta x} \rightarrow \rho$, it is straightforward to see that the first two terms in (26) converge to

$$
\int_{-\infty}^{+\infty} \rho_{0}(x) \varphi(0, x) d x+\int_{0}^{+\infty} \int_{-\infty}^{+\infty} \rho(t, x) \varphi_{t}(t, x) d x d t
$$

as $\Delta x \searrow 0$. Concerning the last term, since $\rho_{j}^{n} \in[0,1]$ we observe that

$$
\begin{aligned}
& \left|g\left(\rho_{j}^{n}, \ldots, \rho_{j+N}^{n}\right)-\rho_{j}^{n} V_{j}^{n}\right| \\
& \leq \frac{\alpha}{2}\left|\rho_{j+1}^{n}-\rho_{j}^{n}\right|+\frac{1}{2}\left|\rho_{j+1}^{n} V_{j+1}^{n}-\rho_{j}^{n} V_{j}^{n}\right| \\
& \leq \frac{\alpha}{2}\left|\rho_{j+1}^{n}-\rho_{j}^{n}\right|+\frac{1}{2}\left|\left(\rho_{j+1}^{n}-\rho_{j}^{n}\right) V_{j+1}^{n}+\rho_{j}^{n}\left(V_{j+1}^{n}-V_{j}^{n}\right)\right| \\
& \leq \frac{1+\alpha}{2}\left|\rho_{j+1}^{n}-\rho_{j}^{n}\right|+\frac{1}{2} \rho_{j}^{n} \Delta x \sum_{k=0}^{N-1} w_{\eta}^{k}\left|\rho_{j+k+1}^{n}-\rho_{j+k}^{n}\right| \\
& \leq \frac{1+\alpha}{2}\left|\rho_{j+1}^{n}-\rho_{j}^{n}\right|+\frac{1}{2} w_{\eta}(0) \operatorname{TV}\left(\rho_{\Delta x}\left(t^{n}, \cdot\right)\right) \Delta x \\
& \leq \frac{1+\alpha}{2}\left|\rho_{j+1}^{n}-\rho_{j}^{n}\right|+C^{\prime}\left(w_{\eta}, \rho_{0}, T\right) \Delta x
\end{aligned}
$$

where we have set $C^{\prime}\left(w_{\eta}, \rho_{0}, T\right)=w_{\eta}(0) C\left(w_{\eta}, \rho_{0}, T\right) / 2$ for $T \geq t^{n}$. Therefore, the last term in (26) can be rewritten as

$$
\begin{aligned}
\Delta & x \Delta t \sum_{n} \sum_{j} \frac{\varphi\left(t^{n}, x_{j+1}\right)-\varphi\left(t^{n}, x_{j}\right)}{\Delta x} g\left(\rho_{j}^{n}, \ldots, \rho_{j+N}^{n}\right) \\
= & \Delta x \Delta t \sum_{n} \sum_{j} \frac{\varphi\left(t^{n}, x_{j+1}\right)-\varphi\left(t^{n}, x_{j}\right)}{\Delta x} \rho_{j}^{n} V_{j}^{n} \\
& +\Delta x \Delta t \sum_{n} \sum_{j} \frac{\varphi\left(t^{n}, x_{j+1}\right)-\varphi\left(t^{n}, x_{j}\right)}{\Delta x} \times\left(g\left(\rho_{j}^{n}, \ldots, \rho_{j+N}^{n}\right)-\rho_{j}^{n} V_{j}^{n}\right) .
\end{aligned}
$$

By consistency, the first term in the above decomposition converges to

$$
\int_{0}^{+\infty} \int_{-\infty}^{+\infty} \rho(t, x) v\left(\rho *_{d} w_{\eta}(t, x)\right) \varphi_{x}(t, x) d x d t
$$

while the second term can be bounded using (27): set $T>0$ and $R>0$ such that $\varphi(t, x)=0$ for $t>T$ and $|x|>R$, and let $n_{T} \in \mathbb{N}$ and $j_{0}, j_{1} \in \mathbb{Z}$ such that $\left.\left.\left.T \in] n_{T} \Delta t,\left(n_{T}+1\right) \Delta t\right],-R \in\right] x_{j_{0}-1 / 2}, x_{j_{0}+1 / 2}\right]$ and $\left.\left.R \in\right] x_{j_{1}-1 / 2}, x_{j_{1}+1 / 2}\right]$, 
then

$$
\begin{aligned}
\Delta x & \Delta t \sum_{n} \sum_{j} \frac{\varphi\left(t^{n}, x_{j+1}\right)-\varphi\left(t^{n}, x_{j}\right)}{\Delta x}\left(g\left(\rho_{j}^{n}, \ldots, \rho_{j+N}^{n}\right)-\rho_{j}^{n} V_{j}^{n}\right) \\
\leq & \Delta x \Delta t\left\|\varphi_{x}\right\|_{\infty} \sum_{n=0}^{n_{T}} \sum_{j=j_{0}}^{j_{1}}\left(\frac{1+\alpha}{2}\left|\rho_{j+1}^{n}-\rho_{j}^{n}\right|+C^{\prime}\left(w_{\eta}, \rho_{0}, T\right) \Delta x\right) \\
= & \frac{1+\alpha}{2}\left\|\varphi_{x}\right\|_{\infty} \Delta x \Delta t \sum_{n=0}^{n_{T}} \sum_{j=j_{0}}^{j_{1}}\left|\rho_{j+1}^{n}-\rho_{j}^{n}\right|+\left\|\varphi_{x}\right\|_{\infty} C^{\prime}\left(w_{\eta}, \rho_{0}, T\right) 2 R T \Delta x \\
\leq & \frac{1+\alpha}{2}\left\|\varphi_{x}\right\|_{\infty} \int_{0}^{T} \int_{-R}^{R}\left|\rho_{\Delta x}(t, x+\Delta x)-\rho_{\Delta x}(t, x)\right| d x d t \\
& +\left\|\varphi_{x}\right\|_{\infty} C^{\prime}\left(w_{\eta}, \rho_{0}, T\right) 2 R T \Delta x \\
\leq & \frac{1+\alpha}{2}\left\|\varphi_{x}\right\|_{\infty} C\left(w_{\eta}, \rho_{0}, T\right) \Delta x+\left\|\varphi_{x}\right\|_{\infty} C^{\prime}\left(w_{\eta}, \rho_{0}, T\right) 2 R T \Delta x
\end{aligned}
$$

which clearly goes to zero when $\Delta x \searrow 0$.

Concerning the entropy condition, we proceed as above to show that $(21)$ converges to (5). Multiplying (21) by $\Delta x \varphi\left(t^{n}, x_{j}\right) \geq 0$ and then summing by parts we get

$$
\begin{aligned}
0 \leq & \Delta x \sum_{j} \varphi\left(0, x_{j}\right)\left|\rho_{j}^{0}-\kappa\right|+\Delta x \Delta t \sum_{n} \sum_{j} \frac{\varphi\left(t^{n}, x_{j}\right)-\varphi\left(t^{n-1}, x_{j}\right)}{\Delta t}\left|\rho_{j}^{n}-\kappa\right| \\
& +\Delta x \Delta t \sum_{n} \sum_{j} \frac{\varphi\left(t^{n}, x_{j+1}\right)-\varphi\left(t^{n}, x_{j}\right)}{\Delta x} F_{j+1 / 2}^{\kappa}\left(\rho_{j}^{n}, \rho_{j+1}^{n}\right) \\
& -\Delta x \Delta t \sum_{n} \sum_{j} \operatorname{sgn}\left(\rho_{j}^{n+1}-\kappa\right) \kappa \frac{V_{j+1}^{n}-V_{j-1}^{n}}{2 \Delta x} \varphi\left(t^{n}, x_{j}\right) .
\end{aligned}
$$

Following the same steps as above, the first three terms in the sum clearly converge to

$$
\begin{aligned}
\int_{0}^{+\infty} & \int_{-\infty}^{+\infty}\left(|\rho(t, x)-k| \varphi_{t}+|\rho(t, x)-k| v\left(\rho *_{d} w_{\eta}(t, x)\right) \varphi_{x}\right) d x d t \\
& +\int_{-\infty}^{+\infty}\left|\rho_{0}(x)-\kappa\right| \varphi(0, x) d x
\end{aligned}
$$

as $\Delta x \searrow 0$. The third term can be decomposed as

$$
\begin{aligned}
& \sum_{n} \sum_{j} \operatorname{sgn}\left(\rho_{j}^{n+1}-\kappa\right) \kappa \frac{V_{j+1}^{n}-V_{j-1}^{n}}{2 \Delta x} \varphi\left(t^{n}, x_{j}\right) \\
= & \left(\operatorname{sgn}\left(\rho_{j}^{n+1}-\kappa\right)-\operatorname{sgn}\left(\rho_{j}^{n}-\kappa\right)\right) \kappa \frac{V_{j+1}^{n}-V_{j-1}^{n}}{2 \Delta x} \varphi\left(t^{n}, x_{j}\right) \\
& +\operatorname{sgn}\left(\rho_{j}^{n}-\kappa\right) \kappa \frac{V_{j+1}^{n}-V_{j-1}^{n}}{2 \Delta x} \varphi\left(t^{n}, x_{j}\right) .
\end{aligned}
$$


The first term in (28) can be controlled by $C\left(w_{\eta}, \rho_{0}\right) \Delta x$, and the second clearly converges to

$$
-\int_{0}^{+\infty} \int_{-\infty}^{+\infty} \operatorname{sgn}(\rho(t, x)-k) k V_{x}(t, x) \varphi(t, x) d x d t,
$$

providing the entropy inequality (5).

\section{Numerical tests}

In this section, we perform numerical simulations to show evidence of some properties of equation (1). In particular, we compare solutions depending on the kernel choice and its support.

For the tests presented below, the space domain is given by the interval $[-1,1]$, the space discretization mesh $\Delta x=0.002$ and $\eta=0.1$, where not specified otherwise. Absorbing conditions are imposed at the boundaries.

\subsection{Kernel support location}

We aim at comparing the behavior of solutions of (1) with centered and upstream convolutions, obtained taking the velocity $v$ as

$$
1-\Delta x \sum_{k=-N / 2}^{N / 2} w_{\eta}^{k} \rho_{j+k} \simeq v\left(\int_{x-\eta / 2}^{x+\eta / 2} \rho(t, y) w_{\eta}(y-x) d y\right),
$$

which models drivers looking both ahead and behind, and

$$
1-\Delta x \sum_{k=-N}^{1} w_{\eta}^{k} \rho_{j+k} \simeq v\left(\int_{x-\eta}^{x} \rho(t, y) w_{\eta}(y-x) d y\right),
$$

corresponding to drivers taking into account traffic densities behind them.

Figure 1 shows similarly the density profiles at time $t=0.5$ corresponding to the initial condition

$$
\rho_{0}(x)= \begin{cases}0.6 & \text { if } x<0 \\ 0.2 & \text { if } x>0\end{cases}
$$

The solution to the classical scalar conservation law

$$
\partial_{t} \rho+\partial_{x}(\rho(1-\rho))=0
$$

would be a rarefaction wave, as displayed in Figure 1, (a).

Figure 2 shows the density profiles at time $t=0.2$ corresponding to the initial condition

$$
\rho_{0}(x)= \begin{cases}0.4 & \text { if } x<0 \\ 0.9 & \text { if } x>0\end{cases}
$$




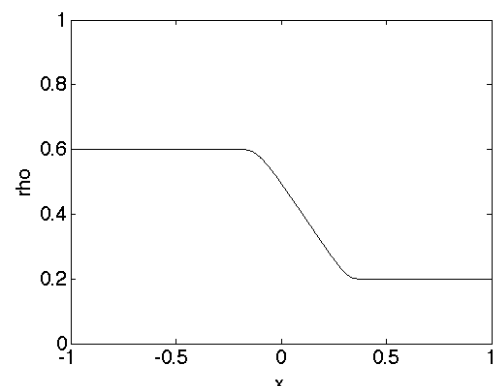

(a)

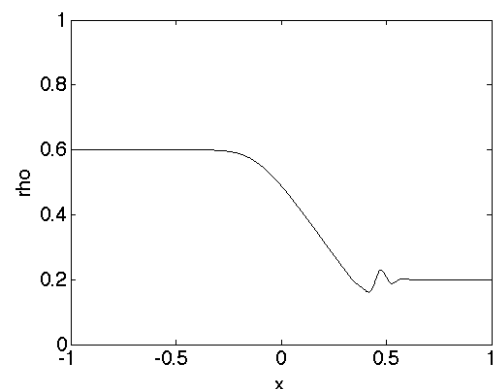

(c)

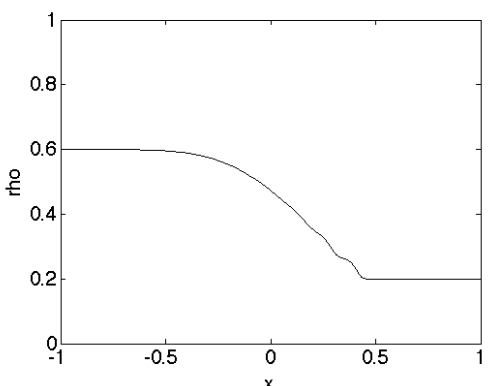

(b)

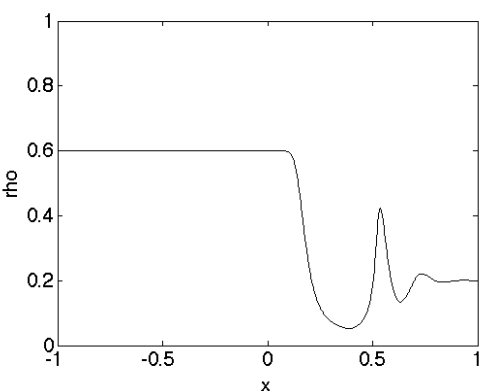

(d)

Fig. 1 Density profiles at $t=0.5$ corresponding to a Riemann-like initial datum with $\rho_{L}=0.6, \rho_{R}=0.2$. Case (a) corresponds to the rarefaction profile that solves the classical equation (32). Cases (b), (c) and (d) correspond to the kernel $w_{\eta}(x)=1 / \eta$ with downstream (2), central (29) and upstream (30) supports respectively.

The solution to the classical scalar conservation law would be a shock traveling with speed $\sigma=-0.3$.

Cases (b), (c) and (d) in Figures 1, 2 show the density profiles corresponding to the constant kernel $w_{\eta}(x)=1 / \eta$ with downstream (2), central (29) and upstream (30) supports respectively. (The approximate solutions do not change significantly using other non-increasing kernels.) We observe that the downstream convolution gives a smooth profile with constant total variation and monotonicity (as expected from Proposition 2) close to the solution of the corresponding classical problem (32) showed in Figures 1, 2, (a). On the contrary, central and upstream convolutions cause oscillations formation and total variation blow-up (for upstream convolution).

\subsection{Kernel monotonicity}

We are interested in studying the effect of the monotonicity of the kernel on the solution characteristics. To this aim, we consider the downstream convolution (2) and we compare the solution corresponding to the constant kernel 


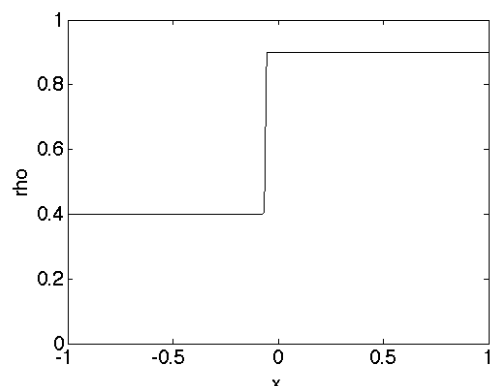

(a)

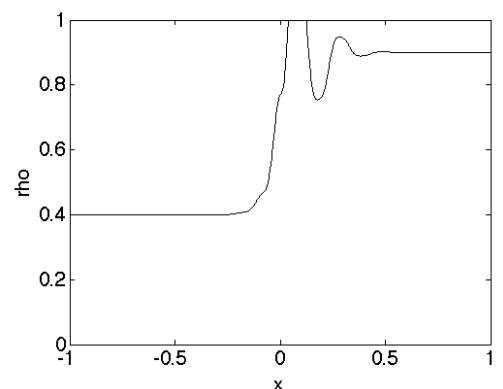

(c)

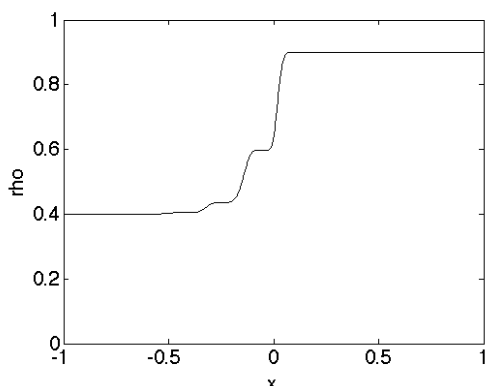

(b)

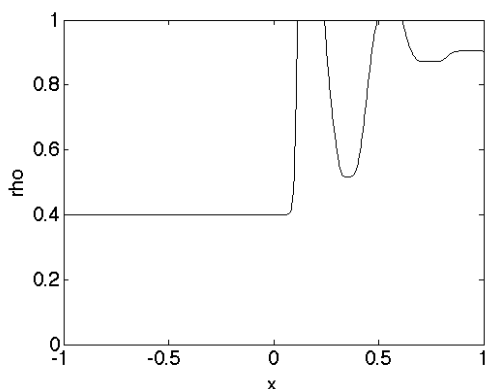

(d)

Fig. 2 Density profiles at $t=0.2$ corresponding to a Riemann-like initial datum with $\rho_{L}=0.4, \rho_{R}=0.9$. Case (a) corresponds to the shock profile that solves the classical equation (32). Cases (b), (c) and (d) correspond to the kernel $w_{\eta}(x)=1 / \eta$ with downstream (2), central (29) and upstream (30) supports respectively.

$w_{\eta}(x)=1 / \eta$, to the linear decreasing kernel $w_{\eta}(x)=2(\eta-x) / \eta^{2}$ and to the linear increasing kernel $w_{\eta}(x)=2 x / \eta^{2}$, for the Riemann-type initial datum (33). Figure 3 shows the density profiles at time $t=0.5$ (left) and the total variation $\operatorname{TV}(\rho(t, \cdot) ;[-1,1])$ for $t \in[0,1]$ (right) corresponding to the different kernel choices. Numerical simulations confirm that non-increasing monotonicity is necessary for the scheme to be monotonicity preserving. In particular, for Riemann type initial data, the total variation remains constant, while it increases for monotonically increasing kernels.

We also compute numerical convergence orders. Following [8], we define

$$
\gamma(\Delta x)=\log _{2}\left(\frac{e(\Delta x)}{e(\Delta x / 2)}\right)
$$

where the $\mathbf{L}^{\mathbf{1}}$-error is computed at final time $T=0.3$ as

$$
\begin{aligned}
e(\Delta x) & =\left\|\rho_{\Delta x}(T, \cdot)-\rho_{\Delta x / 2}(T, \cdot)\right\|_{\mathbf{L}^{1}} \\
& =\frac{\Delta x}{2} \sum_{j}\left|\rho_{\Delta x}\left(T, x_{j}\right)-\rho_{\Delta x / 2}\left(T, x_{j}\right)\right|,
\end{aligned}
$$



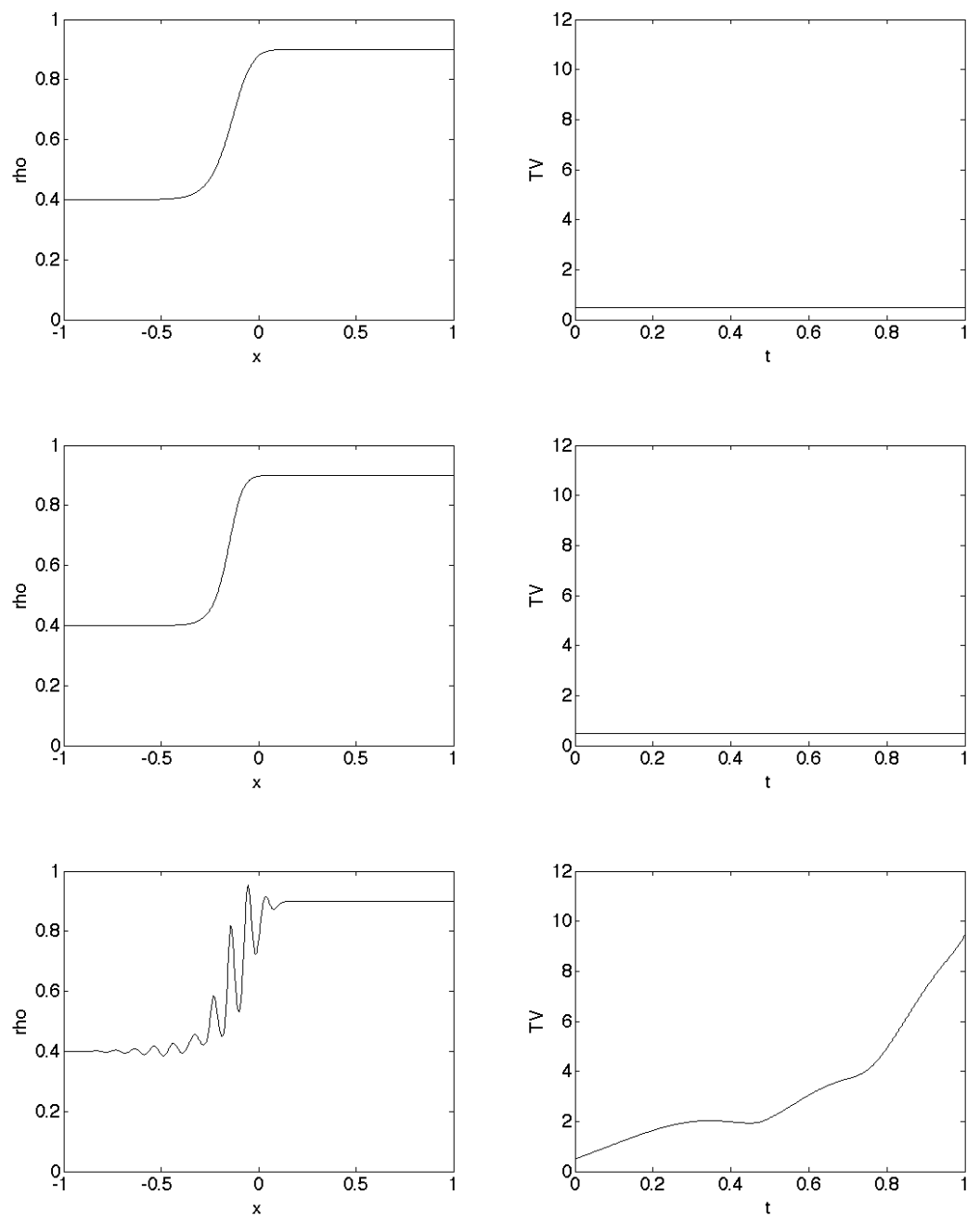

Fig. 3 Density profiles at $t=0.5$ (left) and the total variation $\operatorname{TV}(\rho(t, \cdot) ;[-1,1])$ for $t \in$ $[0,1]$ (right) corresponding to the Riemann-like initial datum with $\rho_{L}=0.4, \rho_{R}=0.9$ for $w_{\eta}(x)=1 / \eta($ top $), w_{\eta}(x)=2(\eta-x) / \eta^{2}$ (middle) and $w_{\eta}(x)=2 x / \eta^{2}$ (bottom).

where $x_{j}=(j-1 / 2) \Delta x / 2$ for $\Delta x=0.01,0.005,0.0025,0.00125,0.000625$. Table 1 show that non-increasing kernels give good convergence rates (the rate is far better for the linear decreasing kernel), while in the case of linear increasing kernel convergence is not clearly established. The $\mathbf{L}^{\mathbf{1}}$-errors reported in the table represent the $\mathbf{L}^{\mathbf{1}}$-distances to the reference solutions corresponding to $\Delta x=0.00015625$. 


\begin{tabular}{|c|c|c|c|c|c|c|}
\hline & \multicolumn{2}{|c|}{$w_{\eta}(x)=1 / \eta$} & \multicolumn{2}{c|}{$w_{\eta}(x)=2(\eta-x) / \eta^{2}$} & \multicolumn{2}{c|}{$w_{\eta}(x)=2 x / \eta^{2}$} \\
\hline$\Delta x$ & $\gamma(\Delta x)$ & $\mathbf{L}^{1}$-error & $\gamma(\Delta x)$ & $\mathbf{L}^{1}$-error & $\gamma(\Delta x)$ & $\mathbf{L}^{1}$-error \\
\hline 0.01 & 0.98021 & $3.013 \mathrm{e}-03$ & 1.06427 & $3.315 \mathrm{e}-02$ & -0.42189 & $1.241 \mathrm{e}-01$ \\
\hline 0.005 & 0.93000 & $1.709 \mathrm{e}-03$ & 1.06119 & $1.590 \mathrm{e}-02$ & -0.88509 & $1.287 \mathrm{e}-01$ \\
\hline 0.0025 & 0.61590 & $1.044 \mathrm{e}-03$ & 0.87964 & $7.650 \mathrm{e}-03$ & -0.13054 & $1.303 \mathrm{e}-01$ \\
\hline 0.00125 & 0.44360 & $6.344 \mathrm{e}-04$ & 1.05856 & $3.696 \mathrm{e}-03$ & 0.15360 & $1.069 \mathrm{e}-01$ \\
\hline 0.000625 & 0.57113 & $3.632 \mathrm{e}-04$ & 0.99995 & $1.547 \mathrm{e}-03$ & 0.27699 & $7.093 \mathrm{e}-02$ \\
\hline
\end{tabular}

Table 1 Convergence orders (34) and $\mathbf{L}^{\mathbf{1}}$-errors to the reference solutions corresponding to $\Delta x=0.00015625$ for constant, linear decreasing and linear increasing kernels at final time $T=0.5$ corresponding to a Riemann-like initial datum with $\rho_{L}=0.4, \rho_{R}=0.9$.

\subsection{Limit $\eta \searrow 0$}

In this section, we investigate the convergence to the solutions of the classical conservation law (32) as $\eta \searrow 0$. To this end, we compare the solutions to (1) with different values of $\eta=0.1,0.01,0.001$, for $\Delta x=0.001$ and initial datum (33). As expected, the solution computed with $\eta=0.001$ coincides with the numerical solution of (1) computed using the Lax-Friedrichs type scheme

$$
\rho_{j}^{n+1}=\rho_{j}^{n}+\frac{\lambda \alpha}{2}\left(\rho_{j-1}^{n}-2 \rho_{j}^{n}+\rho_{j+1}^{n}\right)+\frac{\lambda}{2}\left(f\left(\rho_{j-1}^{n}\right)-f\left(\rho_{j+1}^{n}\right)\right) .
$$

In fact, when $\eta=\Delta x, N=1$ in (7) and the scheme (10), (11) coincides with (36).

4.4 Highly oscillating initial datum

We consider the following initial datum

$$
\rho_{0}(x)= \begin{cases}0.5(1+\sin (10 \pi x)) & \text { if } x \in]-0.5,0.5[ \\ 0.5 & \text { otherwise }\end{cases}
$$

see Figure 5.

We are interested in exploring the effect of the location of the support of $w_{\eta}(x)=1 / \eta$ on the total variation of the solution. Figure 6 shows the density profiles at $t=0.5$ (left) and the total variation $\operatorname{TV}(\rho(t, \cdot) ;[-1,1])$ for $t \in[0,0.5]$ (right). We observe that the downstream convolution (2) smooths down the solution towards the constant profile $\rho(t, x)=0.5$ for $t \rightarrow \infty$. The central convolution (29) still reduces oscillations, but the upstream convolution induces blow up both in the $\mathbf{L}^{\infty}$ and in the BV norms.

Acknowledgements The authors wish to thank Rinaldo M. Colombo and Frédéric Lagoutière for valuable discussions. 

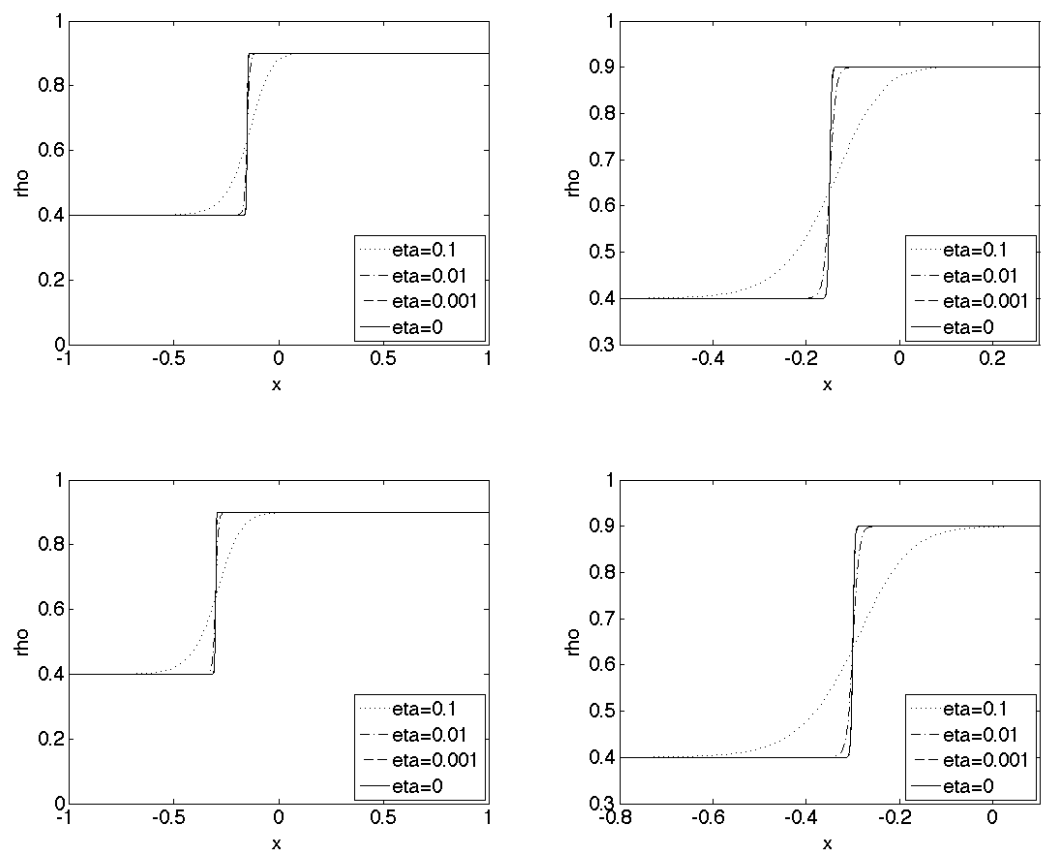

Fig. 4 Density profiles at $t=0.5$ (top) and and $t=1$ (bottom) corresponding to the Riemann-like initial datum with $\rho_{L}=0.4, \rho_{R}=0.9$ for $w_{\eta}(x)=1 / \eta$ and $\eta=0.1,0.01,0.001$.

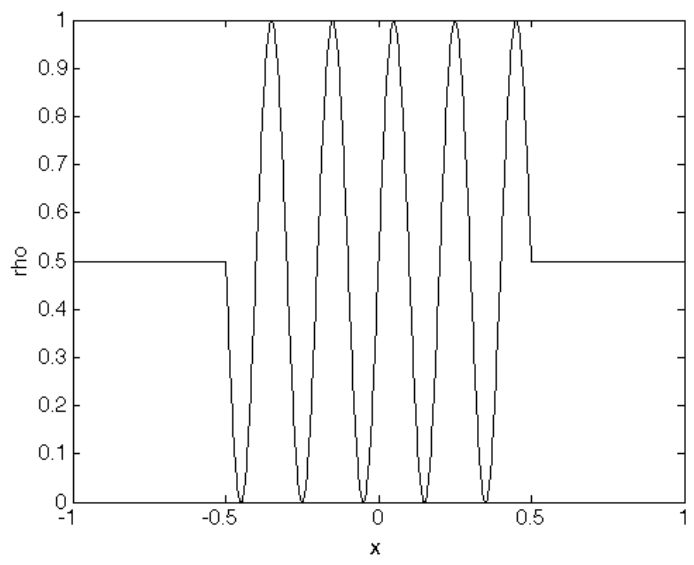

Fig. 5 Oscillating initial datum $\rho_{0}$ given by (37)

\section{References}

1. Amadori, D., Shen, W.: An integro-differential conservation law arising in a model of granular flow. J. Hyperbolic Differ. Equ. 9(1), 105-131 (2012)

2. Amorim, P.: On a nonlocal hyperbolic conservation law arising from a gradient constraint problem. Bull. Braz. Math. Soc. (N.S.) 43(4), 599-614 (2012) 

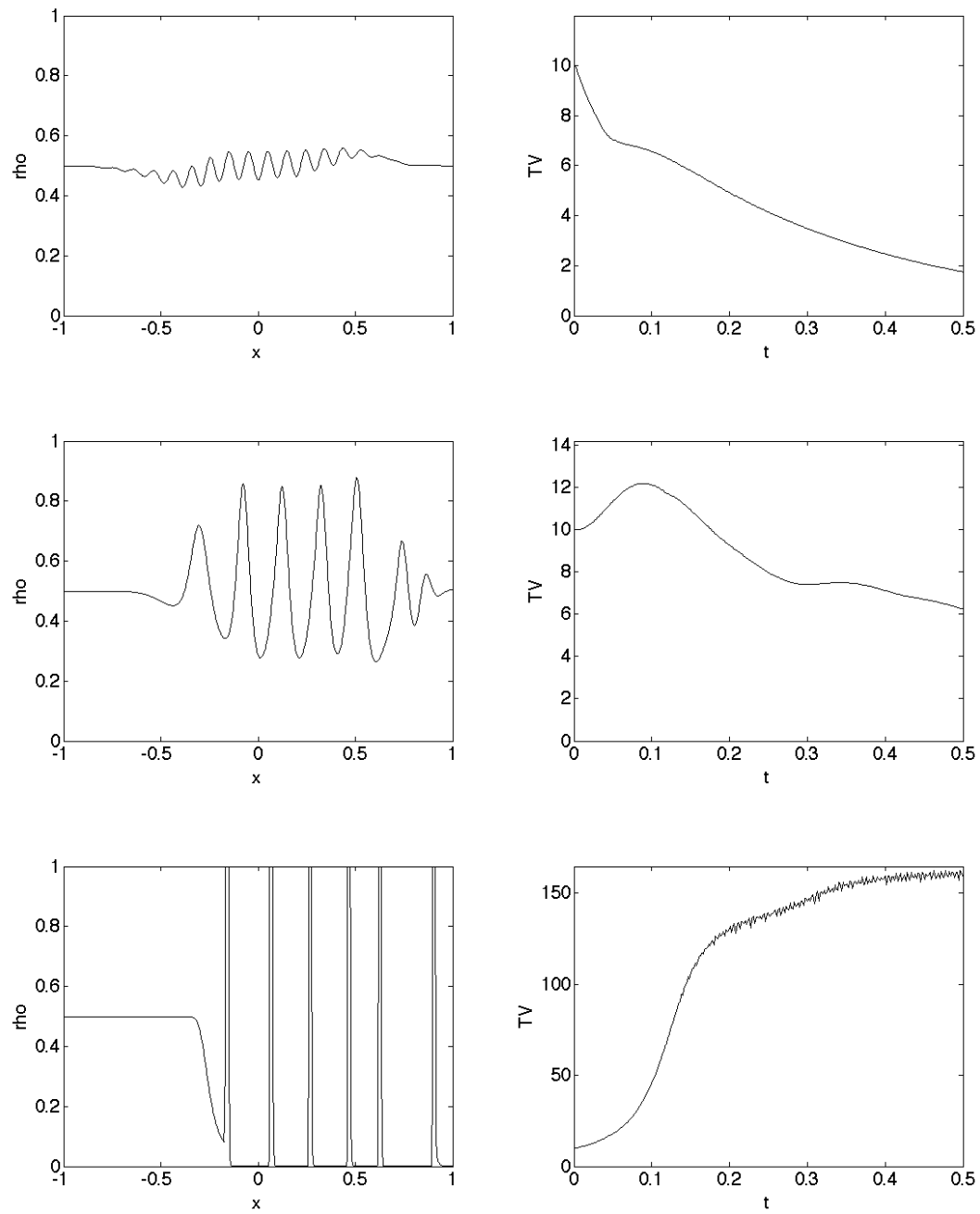

Fig. 6 Density profiles at $t=0.5$ (left) and the total variation $\operatorname{TV}(\rho(t, \cdot) ;[-1,1])$ for $t \in$ $[0,0.5]$ (right) corresponding to the initial datum (37) for $w_{\eta}(x)=1 / \eta$ with downstream (top), central (middle) and upstream (bottom) supports.

3. Amorim, P., Colombo, R.M., Teixeira, A.: A numerical approach to scalar nonlocal conservation laws (2013). Preprint

4. Aw, A., Rascle, M.: Resurrection of "second order" models of traffic flow. SIAM J. Appl. Math. 60, 916-938 (2000)

5. Barth, T., Ohlberger, M.: Finite Volume Methods: Foundation and Analysis. John Wiley \& Sons, Ltd (2004)

6. Betancourt, F., Bürger, R., Karlsen, K.H., Tory, E.M.: On nonlocal conservation laws modelling sedimentation. Nonlinearity 24(3), 855-885 (2011)

7. Blandin, S., Work, D., Goatin, P., Piccoli, B., Bayen, A.: A general phase transition model for vehicular traffic. SIAM Journal on Applied Mathematics. 71(1), 107-127 (2011) 
8. Bretti, G., Natalini, R., Piccoli, B.: Numerical algorithms for simulations of a traffic model on road networks. J. Comput. Appl. Math. 210(1-2), 71-77 (2007)

9. Colombo, R.M., Garavello, M., Lécureux-Mercier, M.: A class of nonlocal models for pedestrian traffic. Mathematical Models and Methods in Applied Sciences 22(04), $1150,023(2012)$

10. Colombo, R.M., Herty, M., Mercier, M.: Control of the continuity equation with a non local flow. ESAIM Control Optim. Calc. Var. 17(2), 353-379 (2011)

11. Colombo, R.M., Lécureux-Mercier, M.: Nonlocal crowd dynamics models for several populations. Acta Math. Sci. Ser. B Engl. Ed. 32(1), 177-196 (2012)

12. Crippa, G., Lécureux-Mercier, M.: Existence and uniqueness of measure solutions for a system of continuity equations with non-local flow. Nonlinear Differential Equations and Applications NoDEA pp. 1-15 (2012)

13. Dafermos, C.M.: Solutions in $L^{\infty}$ for a conservation law with memory. In: Analyse mathématique et applications, pp. 117-128. Gauthier-Villars, Montrouge (1988)

14. Eymard, R., Gallouët, T., Herbin, R.: Finite volume methods. In: Handbook of numerical analysis, Vol. VII, Handb. Numer. Anal., VII, pp. 713-1020. North-Holland, Amsterdam (2000)

15. Harten, A.: High resolution schemes for hyperbolic conservation laws. J. Comput. Phys. 49(3), 357-393 (1983)

16. Kružkov, S.N.: First order quasilinear equations with several independent variables Mat. Sb. (N.S.) 81 (123), 228-255 (1970)

17. Lebacque, J.P., Mammar, S., Salem, H.H.: Generic second order traffic flow modelling. In: International Symposium on Transportation and Traffic Theory 2007 (2007)

18. LeVeque, R.J.: Numerical methods for conservation laws, second edn. Lectures in Mathematics ETH Zürich. Birkhäuser Verlag, Basel (1992)

19. Lighthill, M.J., Whitham, G.B.: On kinematic waves. II. A theory of traffic flow on long crowded roads. Proc. Roy. Soc. London. Ser. A. 229, 317-345 (1955)

20. Richards, P.I.: Shock waves on the highway. Operations Res. 4, 42-51 (1956)

21. Sopasakis, A., Katsoulakis, M.A.: Stochastic modeling and simulation of traffic flow: asymmetric single exclusion process with Arrhenius look-ahead dynamics. SIAM J. Appl. Math. 66(3), 921-944 (electronic) (2006) 